\title{
A Metamodel for Designing an Intelligent Tutoring Systems Authoring Tool
}

\author{
Adetokunbo A. A. Adenowo ${ }^{1,2} \&$ Ashok M. Patel ${ }^{2}$ \\ ${ }^{1}$ Department of Electronic \& Computer Engineering, Lagos State University, Lagos, Nigeria \\ ${ }^{2}$ CAL Research \& Software Engineering Centre, De Montfort University, Leicester, United Kingdom \\ Correspondence: Adetokunbo A. A. Adenowo, Department of Electronic \& Computer Engineering, Lagos State \\ University, Lagos, Nigeria. E-mail: adetokyom@yahoo.com
}

\author{
Received: March 2, 2014 Accepted: March 31, 2014 Online Published: April 28, 2014 \\ doi:10.5539/cis.v7n2p82 URL: http://dx.doi.org/10.5539/cis.v7n2p82
}

\begin{abstract}
Previous intelligent tutoring systems (ITS) and ITS authoring studies predominantly simulated and evaluated artificial intelligence (AI) techniques and cognitive architectures/notions in educational domains. Current research focuses on software design that is priori driven by educational theories; it concerns the conception of Augmented Conversation and Cognitive Apprenticeship Metamodel (ACCAM). The pedagogy driven metamodel-ACCAM - forms the basis for a formal (theory based) approach to designing ITS authoring tools for numerical aspect of numerical disciplines. This research, therefore, showcases the convergence of two theoretical perspectives - the Conversation Theory (CT) and Cognitive Apprenticeship (CA) — which were never considered together before now. The novel conceptual platform-the ACCAM-flows and benefited from the synergistic effect of the stated theories through the introduction of the concept of 'augmented conversation' within the resulting integrated framework. Thus, current work draws on the pedagogical import of the mentioned educational theories, elicits new meanings, and lays the foundation as well as opens future evaluation of a pedagogical engineering methodology that flows therefrom.
\end{abstract}

Keywords: metamodel, intelligent tutoring system, ITS authoring, augmented conversation, conversation theory, cognitive apprenticeship

\section{Introduction}

This work contributes conceptually - through the conception of Augmented Conversation and Cognitive Apprenticeship Metamodel (ACCAM) - to a large body of research on intelligent tutoring systems (ITS) and ITS authoring. Note that an ITS authoring tool, herein also known and referred to as Intelligent Learning Activity Builder (ILABS), is a generalised framework for building ITSs along with a user interface that can be utilised by non-programmers to formalise and visualise their knowledge (Murray, 2003a).

Research in the field of ITS authoring emerged to address problems associated with turning out large number(s) of useful ITSs. Although there are commercial authoring tools, they appear best suited for traditional computer aided instruction (CAI) systems and multimedia-based training because they lack the sophistication to construct ITSs (Murray, 2003a, 2003b). The referred commercial authoring tools excel in providing rich assets that are useful for designing visually appealing and interactive screens, but behind these screens are shallow representations of content and pedagogy strategies (Murray, 2003a). This necessitated specialised authoring tools that address the needs of ITS construction.

In view of the foregoing, previous ITS authoring researches demonstrated varying perspectives. These perspectives include the investigation/implementation of a number of AI techniques and methodologies (e.g. Bayesian networks, neural networks, etc.-Li, Zhuying, \& Bing, 2010; Zarandi, Khademian, \& Minaei-Bidgoli, 2012), cognitive architectures and/or notions (e.g. ACT-R, SOAR, etc.) (Aleven, Sewall, McLaren, \& Koedinger, 2006a; Aleven, McLaren, Sewall, \& Koedinger, 2006b; Blessing, Gilbert, Ourada, \& Ritter, 2009) and human computer interaction standards (Murray, 1998, 2003b). Only few cases lay claim to the investigation of educational theories in ITS (Nkambou, Frasson, \& Gauthier, 2003; Hayashi, Bourdeau, \& Mizoguchi, 2009). The aforementioned techniques/methodologies were used to simulate computational models and to mimic human tutor's intelligence in educational machines (Graesser, Conley, \& Olney, 2012). Despite their relative successes, 
it is arguable that the above stated techniques, architectures and/or notions did not inform the design of intelligent and adaptive educational tools, neither do they appear to translate to theories (Self, 1990, 1994, 1999).

It could be argued that several factors (i.e. intellectual skills, cognitive strategies, verbal information, attitudes, and motor skills) come to play in the teaching and learning domain and educational theories appear to have identified them. It is therefore arguable that a design with a priori link to theory could constitutes a basic requirement that should be satisfied if sound educational tools, that address the needs of diverse learners, are to emerge (Hartley, 2010).

Thus, this paper principally presents a pedagogic (or conceptual) metamodel, ACCAM. The metamodel will shape the design of an ITS authoring tool, a tool meant to generate intelligent tutoring applets for educational support purpose. The conceptual metamodel advances two educational theories, Conversation Theory (CT) (Pask, 1976) and Cognitive Apprenticeship (CA) (Collins, Brown, \& Newman, 1989). The metamodel builds on the theoretical knowledge and assumptions of the aforementioned theories. In addition, the metamodel lays the foundation to investigate twin theoretical concepts, "augmented conversation" and "improved cognitive visibility," in future work. "Augmentation" concept advances current understanding of the existing theories. It aims to enhance the diagnosis of the learning process of a learner through "improved cognitive visibility" of his/her thought process during knowledge construction. Both the former and latter concepts aim the construction of ITSs that supports interventionist strategy. Overall, this conceptual provision aims the construction of ITSs that enhances effective learning.

The rest of the paper is structured as follows: section 2 presents an overview of ITS authoring research, stating the design factors; section 3 provides justification for the conception a pedagogic metamodel; and section 4 examines the theories that underpin the metamodel herein discussed. The conception of ACCAM with its conceptual elements and the design implications of these elements are presented in section 5 and 6 respectively. Finally, section 7 provides a summary/conclusion of the research work, while section 8 presents future work.

\section{Trends in ITS Authoring Tools' Design}

Many of the existing ITS authoring tools appear to lack grounded theoretical or philosophical foundation. This stands to be a requirement to evolve theoretically formalised designs (similar to that proffered in favour of ITS in Self [1990, 1999]) and constitutes the basis to evaluate the learning effectiveness of ITSs that might emerge from a formalised authoring tool. A review of the literature shows that the design of many ITS authoring tools is driven by goals such as usability and reusability, knowledge representation and their pedagogy basis. For instance, Virvou and Moundridou (2000), Brusilovsky (2003), Blessing et al. (2009) and Gilbert, Devasani, Kodavali and Blessing (2011) argued that the usability of ITS authoring tools and reusability of their products (i.e. ITS) should constitute two significant factors that should be considered when designing; otherwise, the purpose of such authoring tools will be defeated. Thus, a design driven by the aforementioned goals could be said to be far away from a formal-based design approach.

As earlier mentioned, two main disciplines-AI and Cognitive Science-have driven and dominated ITS/Authoring research right from inception. In effect, ITS authoring tools reflected the implementation of AI methodologies (see Zarandi, Khademian, \& Minaei-Bidgoli, 2012), and psychological models such as ACT-R cognition model (see Blessing et al., 2009; Aleven et al., 2006a; Aleven et al., 2006b) and constraint-based model (see Mitrovic, Martin, \& Suraweera, 2009). The discipline of Human Computer Interaction (HCI) and existing commercial authoring tools played significant influencing role in the design of ITS authoring tools. HCI contributed in terms of intuitive design and commercial authoring tools and provided features that could be embedded in ITS authoring tools. The impact of these two, HCI and commercial authoring tools, are observable in the design of EON, a pedagogy-oriented ITS authoring tool (Murray, 1998, 2003b).

Some exceptions to the above are ITS authoring tools traceable to educational theories. Tools in this category include: Curriculum REpresentation and Acquisition Model-Tools-CREAM-Tools (Nkambou, Frasson, \& Gauthier, 2003) and SMARTIES (Hayashi, Bourdesau, \& Mizoguchi, 2009). CREAM-Tools are set of tools that allow creation and organisation of a curriculum according to three models: the domain, the pedagogy and the didactic (i.e. resources) aspects. CREAM's design was informed by Gagne's taxonomy (Nkambou, Frasson, \& Gauthier, 2003) - an hierarchy of learned capabilities or types of knowledge (intellectual skills, cognitive strategies, attitudes, and motor skills) required to achieve learning. However, Gagne's taxonomy only shaped one component of the CREAM-Tools, the Knowledge Representation (KR) model. This contrast with current research, in which, all aspects of an authoring tool is conceived and shaped by a theory-based formalisation.

SMARTIES (Hayashi, Bourdeau, \& Mizoguchi, 2009) is designed to be a theory-aware authoring tool; it is linked to an ontology named OMNIBUS (Mizoguchi, Hayashi, \& Bourdeau, 2010). It is considered to be a step 
towards theory-based formalised design because it implements multiple instructional theories and adopts theory-ontology engineering approach (Nkambou, Bourdeau, \& Mizoguchi, 2010). However, SMARTIES lacks specific pedagogical focus due to its multiple theories (i.e. utilised 9 theories). As a result, it requires AI agents to infer the user or author's pedagogy.

Unlike SMARTIES, current research targets a specific pedagogical perspective, ruling out the use of AI techniques and suggests a truly theory-driven design. Thus, this work proposes a pedagogic metamodel that advances two constructivist theoretical frameworks. ITS authoring tools based on the proposed pedagogic metamodel do not need AI-agents to infer author's pedagogy. Also, an ITS authoring tool based on the pedagogic metamodel can generate ITSs implementing the conversation-cognitive approach, unlike SMARTIES. This will enable the monitoring of a learner's cognitive process in order to support his/her learning of a target domain.

\section{Basis for a Pedagogic Metamodel}

ITS authoring tool underpinned by pedagogical assumptions drawn from conventional teaching and learning theories can adequately reflect the pedagogical strategies and elements of its underlying theories. Such an educational approach to design stands to clarify the ontological and epistemological stance of a tool, which may be helpful in furthering and understanding research based on such a tool. It has the advantage of predicting the possible learning process of its product. Likewise, it can help decision-making, especially, when choosing among tools that can support traditional teaching and learning processes. The latter point is critical, in that a purchaser of an instructional support tool might ask: "what is really available (or soon to be available) to make ITS authoring cost effective?" (Murray, 2003a, p. 492). Also, it could help tailor ITS construction towards the teaching and learning goals of parent authoring tool, contrast to the trend in the literature in which new AI implementations or cognitive computational models are tested or simulated.

Furthermore, formalisation of ITS authoring tools using educational theories benefits from the rich theoretical foundation of the education field. It can inform a good design since many of the conventional educational theories - such as CT (Pask, 1976) and CA theory (Collins, Brown, \& Newman, 1989) - capture the teaching-learning variables applicable in a technology-enhanced learning environment. For example, theories could: inform appropriate learning context/settings, identify features that enhance and/or inhibit teaching-learning process, inform appropriate knowledge representation (KR) scheme and strategies for implementing learning content and feedback scaffolding.

Theories stand to be valuable conceptual frameworks for the design of useful ITS authoring tools. More so, some elements of these educational theories (e.g. scaffolding, fading etc.) have been mentioned in some ITS/Authoring tools (thus indicating their significance), but without the formal implementation of these theories. Examples are WEAR - WEB based authoring tool for Algebra Related domains (Moundridou \& Virvou, 2001, 2002, 2003), and Advanced Geometry Tutor (Matsuda \& VanLehn, 2005). The question is, "if these conceptual variables are valuable to these tools, then why not formalise the design of these tools using educational theories since the variables emerged from these pedagogical theories?" This is an open question that queries the intent behind their use in previous researches. This contributed to the idea or need to formalise the design of ITS authoring tools using educational theories. Such formalisation should be a priori and implemented in such way that generates relevant and useful ITSs. It would provide a window to test the applicability of an educationally theory driven metamodel design (Adenowo, 2012).

\section{ACCAM: The Underlining Theories}

As earlier stated, the pedagogic metamodel (i.e. ACCAM) is underpinned by two conventional theories $-\mathrm{CT}$ and $\mathrm{CA}$. These theories contribute to ACCAM in terms of philosophical stance, teaching-learning context and other conceptual elements - mentioned later. The elements will inform the design features of an ITS authoring tool underpinned by the pedagogic metamodel. For the first time, this work integrates the existing theoretical frameworks, the conversation theory and cognitive apprenticeship, and elicits new meanings through the augmented conversation taking place within an integrated framework. The augmentation is conceptualised as an interactive medium of conversation through which the thought process of a learner in a pedagogic process can be expressed and monitored. Below, the above mentioned theories - CT and CA-are presented.

\subsection{Conversation Theory (CT): A Cursory Overview}

CT is a theory of learning and teaching (Scott \& Cong, 2010) that was propounded by Gordon Pask (Pask, 1975). Although the theory has found real world use in education (Scott, 2007), it is also considered a cybernetic theory due to its origin from a cybernetic and dialectic framework (Scott, 2001b; Sharples, 2005). CT elucidates how interactions between participants in a conversation lead to the "construction of knowledge" (Scott \& Cong, 2008) 
and promotes a "radical constructivist" epistemology of human learning (Scott, 2001b).

According to CT, conversation involves two or more participants, the 'psychological (p-) individuals' - coherent conceptual systems - embodied in 'mechanical ( $\mathrm{m}$-) individuals' (processors, brains, bodies and argumentations) (Scott \& Cong, 2008). The theory distinguishes the stable and self-reproducing systems, namely the P-individuals and M-individuals. $P$-individual - a self-reproducing class of procedures - is executable in one or more restricted class of $M$-individuals (Pask, Scott \& Kallikourdis, 1973; Boyd, 2004). CT emphasises two elements: a medium of knowledge construction - termed the "conversation"-and a student - the "knower"-within a communication space.

Also, the theory recognises the existence of distinct domains of knowledge. The distinction between these domains is subject to negotiation and agreement within the conversations, constituted by a community of observers (i.e. participants/individuals in a conversation). CT suggests a methodology-knowledge and task analysis - for analysing the structure of different knowledge domains. Being a whole theory, CT is considered a framework for understanding knowledge and reality, and embodies the epistemology and methodology for investigating the world of objective reality.

\subsection{Cognitive Apprenticeship (CA): A Cursory Overview}

On the other hand, CA promotes a socio-constructivist perspective to human learning. The theory advocates an apprenticeship approach to learning, where a master-apprentice relationship is established in a situated context to achieve successful learning (Collins, Brown, \& Newman, 1989; Dennen \& Burner, 2008). Cognition is regarded a key player in a CA-driven learning process which takes place in a social context, and learning is conceived to commence from activity to abstraction.

Contrast to traditional apprenticeship — wherein learning is external and bears a concrete product, cognitive skill is internal (or hidden). Cognitive skill needs to be open so that participants in the social learning process can support one another in knowledge construction. CA approach aims to open-up the tacit cognitive processes in participants so that the learner can observe, enact, and practice with help from others in the social learning space (Collins, Brown, \& Holum, 1991). The process of opening-up the tacit processes running in learner and master is known as cognitive visibility.

In order to achieve cognitive visibility, the theory suggests six methods (modelling, coaching, scaffolding, articulation, reflection and exploration) which are discussed below. In this paper, the theory contributes methodologically and informs the concept of "improved cognitive visibility" in the proposed metamodel.

\section{ACCAM: Underlining Philosophy and Conceptual Elements}

The philosophical stance and theoretical knowledge and assumptions of above discussed theories clarify how knowledge and skills evolve and advance. The theories' conceptual elements introduce theoretical and pedagogical issues believed to be apt and significant to stimulate the development of an educational tool. ACCAM brings together the theoretical features of these theories, reorganises and augments the conversation element therein. While the metamodel shares the common grounds of the theories; it also builds on their divergent areas and pays attention to the theoretical implications of their synthesised effects, as illustrated in figure 1 below.

Consequently, ACCAM evolves a philosophical stance, a synthesised abstraction of the philosophical positions of the two constructivist theories that underpin it. The synthesis brings to the fore an epistemological and ontological position that underpins the metamodel. Table 1 below presents ACCAM's philosophical position and other theoretical elements-learning content, KR scheme, content sequencing strategies and pedagogy methods including the conversation strategy and the social context of learning - which are derived from the synthesised theoretical frameworks. The table highlights the source(s), meaning and implication(s) of the metamodel's elements. Thereafter, a detailed discussion is rendered which presents the standpoints of the metamodel with respect to its constituting conceptual elements. Figure 2 below presents the proposed teaching and learning process that flows from the metamodel.

It should be noted that conversation, the pedagogy medium of $\mathrm{CT}$, is augmented as an element of the metamodel; it can be internal (i.e. within the P-individual through negotiation of internal processes) or external (i.e. between M-individuals - two cognitive systems). Also, conversation is conceived in the metamodel to occur in three phases within applicable CA methods, grouped as follows: phase 1-involves modelling (including explanation), coaching and scaffolding (including fading); phase 2-includes reflection and articulation; and phase 3 - involves exploration. 


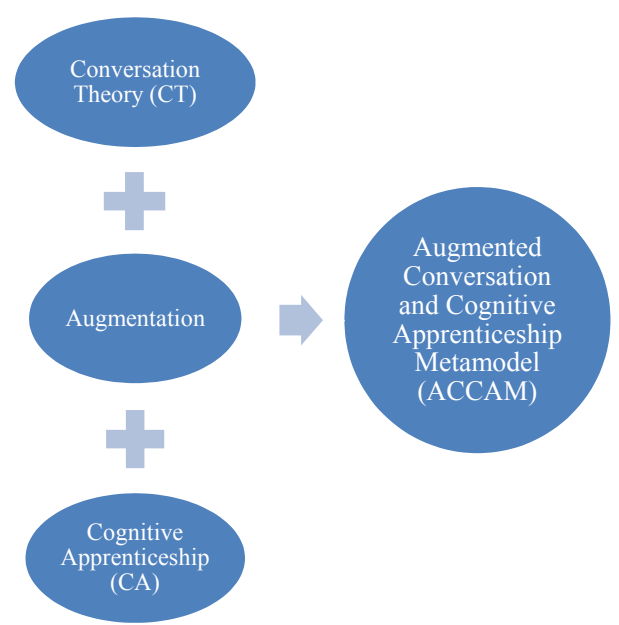

Figure 1. Evolution of ACCAM from CT \& CA

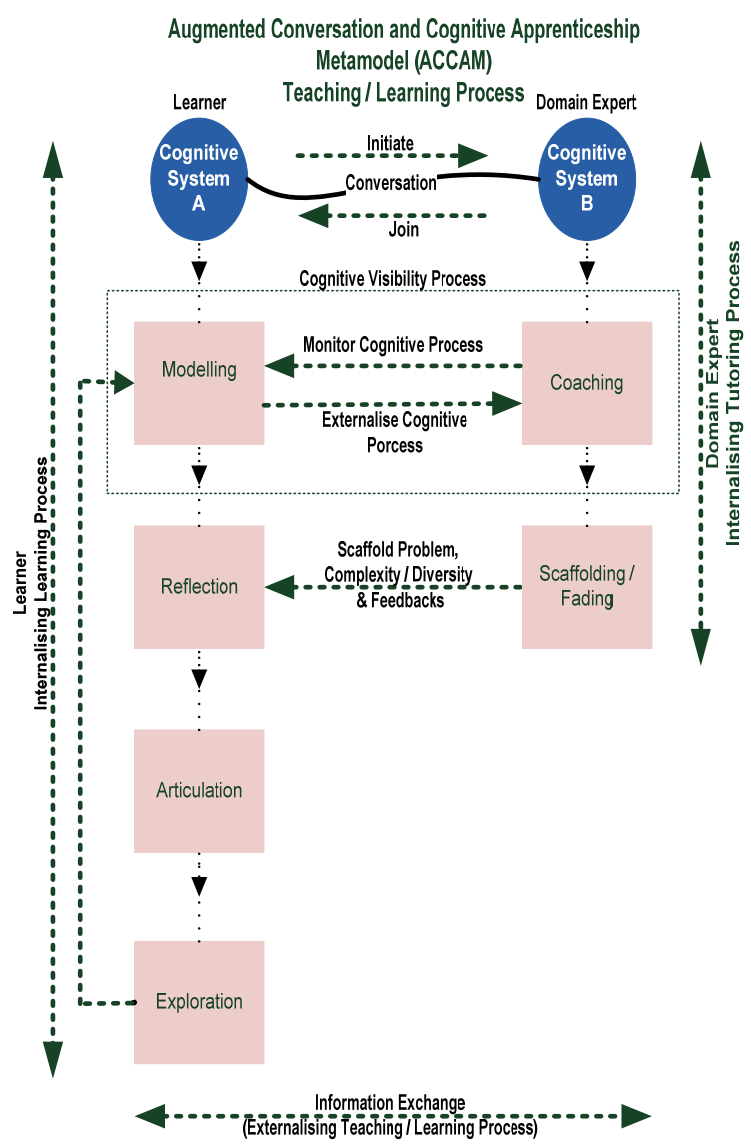

Figure 2. The ACCAM pedagogy process 
Table 1. Synthesised theoretical elements of ACCAM

\begin{tabular}{|c|c|c|c|}
\hline Theoretical Elements & $\begin{array}{l}\text { Conversation Theory } \\
\text { (CT) }\end{array}$ & $\begin{array}{c}\text { Cognitive } \\
\text { Apprenticeship (CA) }\end{array}$ & $\begin{array}{l}\text { Augmented Conversation \& Cognitive } \\
\text { Apprenticeship Metamodel (ACCAM) }\end{array}$ \\
\hline $\begin{array}{l}\text { Nature of knowledge: } \\
\text { Epistemological/Ontolo } \\
\text { gical stance or } \\
\text { assumption. }\end{array}$ & $\begin{array}{l}\text { Knowledge exists and } \\
\text { evolves, and is } \\
\text { advanced through } \\
\text { conversation, } \\
\text { represented by } \\
\text { entailment structures. }\end{array}$ & $\begin{array}{l}\text { Knowledge evolves when } \\
\text { situated and advanced } \\
\text { through socio-interaction. }\end{array}$ & $\begin{array}{l}\text { Knowledge exists, evolves and advances } \\
\text { when situated through socio-interactive } \\
\text { conversations with a minimum of two } \\
\text { participants. } \\
\text { Learning involves establishing } \\
\text { relationships among the concepts of the } \\
\text { subject matter. } \\
\text { Knowledge is structured as a network of } \\
\text { interrelated concepts captured as } \\
\text { entailment structures. }\end{array}$ \\
\hline Content & $\begin{array}{l}\text { Conceived as the } \\
\text { subject domain upon } \\
\text { which learning is based } \\
\text { and includes topics and } \\
\text { their concepts and task } \\
\text { structures. }\end{array}$ & $\begin{array}{l}\text { Conceived as both the } \\
\text { subject domain and the } \\
\text { three strategies deployed } \\
\text { during learning. } \\
\text { Domain knowledge must } \\
\text { be situated or tailored to } \\
\text { practice. }\end{array}$ & $\begin{array}{l}\text { Consists of domain knowledge, captured } \\
\text { as entailment structures of } \\
\text { topics/concepts, yet situated in practice. } \\
\text { Assumes CA strategies, but enhanced to } \\
\text { remove conversation uncertainties } \\
\text { (termed cognitive 'fixity' by Pask). }\end{array}$ \\
\hline $\begin{array}{l}\text { Strategies } \\
\text { Protocols }\end{array}$ & $\begin{array}{l}\text { Deployed to remove } \\
\text { conversation } \\
\text { uncertainties, termed } \\
\text { cognitive 'fixity' by } \\
\text { Pask, a situation that } \\
\text { occurs when habits of } \\
\text { action-old learning } \\
\text { habits-block new } \\
\text { learning. }\end{array}$ & Part of content & $\begin{array}{l}\text { This element assumes CT's definition, } \\
\text { but considered in ACCAM as part of } \\
\text { domain content. }\end{array}$ \\
\hline $\begin{array}{l}\text { Entailment and } \\
\text { entailment structures }\end{array}$ & $\begin{array}{l}\text { Represent a KR scheme } \\
\text { for domain knowledge. }\end{array}$ & Not prescribed. & This element assumes CT's definition. \\
\hline Participants & $\begin{array}{l}\text { Conceived as integrated } \\
\text { distributed cognitive } \\
\text { systems, i.e. } \\
\text { M-Individuals and/or } \\
\text { P-individual(s), } \\
\text { depending on the } \\
\text { number of internal } \\
\text { processes occurring in } \\
\text { the individual's brain } \\
\text { during learning. } \\
\text { Must include a domain } \\
\text { expert and learner(s). } \\
\text { Minimum of two } \\
\text { participants involved. }\end{array}$ & $\begin{array}{l}\text { Conceived as individuals } \\
\text { participating in } \\
\text { socio-interaction learning. } \\
\text { Must include both } \\
\text { experienced person(s) and } \\
\text { less experienced person(s) } \\
\text { in a master-apprenticeship } \\
\text { relationship. } \\
\text { Minimum of two } \\
\text { participants involved. }\end{array}$ & $\begin{array}{l}\text { Assumed CT meaning with the integrated } \\
\text { cognitive systems and/or P-individuals, } \\
\text { which embodies the socio-interaction } \\
\text { learning context of CA. } \\
\text { Must include a domain expert (or master) } \\
\text { and learner(s). } \\
\text { Minimum of two participants involved. }\end{array}$ \\
\hline Procedures & $\begin{array}{l}\text { A set of synchronised } \\
\text { programs-usually } \\
\text { nondeterministic or } \\
\text { fuzzy algorithms that } \\
\text { coordinate conversation. }\end{array}$ & Not prescribed. & $\begin{array}{l}\text { Included as defined by CT, but } \\
\text { implemented as "pure" algorithm (i.e. } \\
\text { algorithm that is AI-neutral, instead, } \\
\text { interface-based). }\end{array}$ \\
\hline
\end{tabular}




\begin{tabular}{|c|c|c|c|}
\hline Theoretical Elements & $\begin{array}{l}\text { Conversation Theory } \\
\text { (CT) }\end{array}$ & $\begin{array}{c}\text { Cognitive } \\
\text { Apprenticeship (CA) }\end{array}$ & $\begin{array}{l}\text { Augmented Conversation \& Cognitive } \\
\text { Apprenticeship Metamodel (ACCAM) }\end{array}$ \\
\hline Formal language & $\begin{array}{l}\text { The medium or } \\
\text { language of } \\
\text { conversation agreed to } \\
\text { by all participants. } \\
\text { This should occur in at } \\
\text { least three levels to } \\
\text { avoid cognitive 'fixity'. }\end{array}$ & $\begin{array}{l}\text { Observation, enactment } \\
\text { and practising. }\end{array}$ & $\begin{array}{l}\text { Informal or non-verbal conversation, or } \\
\text { what Holland \& Childress (2008) regard } \\
\text { as information exchange between learner } \\
\text { and domain expert, involving active } \\
\text { participation (e.g. practising). }\end{array}$ \\
\hline Environment & $\begin{array}{l}\text { Represents the } \\
\text { conversation machine(s) } \\
\text { and interfaces that } \\
\text { facilitate externalisation } \\
\text { of multilevel } \\
\text { conversations (i.e. } \\
\text { information exchange or } \\
\text { bi-directional } \\
\text { communication) among } \\
\text { participants-similar to } \\
\text { pencil and paper, chalk } \\
\text { and blackboard etc. }\end{array}$ & Not prescribed. & $\begin{array}{l}\text { Included as defined in CT. } \\
\text { Deployed to make visible inner processes } \\
\text { taking place in individual participants as } \\
\text { required in CA. }\end{array}$ \\
\hline Social Context & $\begin{array}{l}\text { Learning takes place in } \\
\text { an informal social } \\
\text { context. }\end{array}$ & $\begin{array}{llr}\text { Learning takes } & \text { place } \\
\text { explicitly in a } & \text { social } \\
\text { context. } & & \\
\text { lat }\end{array}$ & $\begin{array}{l}\text { Assumes both informal and formal social } \\
\text { contexts, but limited to two participants } \\
\text { (in this implementation). }\end{array}$ \\
\hline $\begin{array}{l}\text { Teaching and learning } \\
\text { Methods }\end{array}$ & $\begin{array}{l}\text { Prescribes 'teachback' } \\
\text { strategy to facilitate } \\
\text { understanding and } \\
\text { knowledge transfer. }\end{array}$ & $\begin{array}{l}\text { Prescribes six } \\
\text { methods-modelling, } \\
\text { coaching, scaffolding, } \\
\text { articulation, reflection and } \\
\text { exploration-for moving } \\
\text { a learner from actual state } \\
\text { (low end) to expected } \\
\text { state (high end). }\end{array}$ & $\begin{array}{l}\text { Assumes conversation that involves } \\
\text { seven methods of pedagogy (CA methods } \\
\text { and CT 'teachback'). } \\
\text { Assumes three concepts: Zone of } \\
\text { Proximal Development (ZPD), } \\
\text { 'intersubjectivity' and fading as part of a } \\
\text { scaffolding method. Intersubjectivity } \\
\text { refers to having shared understanding or } \\
\text { goal, lack of which is evident in learning } \\
\text { conflict among participants in a learning } \\
\text { situation (Dennen, 2004) }\end{array}$ \\
\hline Sequencing & $\begin{array}{l}\text { Sequencing of subject } \\
\text { matter materials are } \\
\text { based on the learning } \\
\text { style of a learner. Not } \\
\text { just a sequence from } \\
\text { global to specific. }\end{array}$ & $\begin{array}{l}\text { Promotes sequencing of } \\
\text { learning activity from } \\
\text { global to specific. }\end{array}$ & $\begin{array}{l}\text { Integrated both ideas from both theories. } \\
\text { Identified the need to recognise the } \\
\text { different learning styles of a learner. } \\
\text { Despite that, still recognises the need to } \\
\text { sequence learning materials in ways that } \\
\text { promote understanding irrespective of } \\
\text { each learner's style. }\end{array}$ \\
\hline
\end{tabular}

\subsection{The Philosophical Stance-Epistemological/Ontological Positions}

The teaching and learning theories range between two extremes of an axis (von Glasersfeld, 2002). Whatever is proposed by one end of the axis has element(s) of the other end of the axis, and could be demolished by grounded arguments (von Glasersfeld, 2002). This informed the need to clarify the philosophical position of ACCAM since it is meant to shape tools deployed in a technology-enhanced learning (TEL) environment. Philosophically, ACCAM assumes three key concepts, namely existence, evolvement and advancement of knowledge. On epistemologically ground, the metamodel holds that knowledge exists, evolves and progresses within individual participants (Pask, 1988) or through reflection (Collins, Brown, \& Newman, 1989), as well as through interaction of participants (Pask, 1975; Collins, Brown, \& Newman, 1989) when knowledge is situated in practice (Collins, Brown, \& Newman, 1989). Pask (1976) argued that this interaction could be a network of cognitive systems, while Collins, Brown and Newman (1989) explicitly based it on sociological interaction. Implicitly, Pask's cognitive systems interaction can be said to be a socio-interaction since participants behind the cognitive systems could be human (the learners) or organisations. 
The foregoing stance assumes a numerical problem solving context (the focus and context of ACCAM's implementation) in which domains are practice-based or procedurally-oriented. Generally, domain knowledge comprises of declarative and procedural knowledge (Ohlsson \& Mitrovic, 2006; Akin, 1986, 2008). Learning in a numerate domain requires initial romance with the declarative aspect of knowledge (the first step in Whitehead's learning cycle-see Boyd, 2004). The romance takes precedence over meaningful procedural learning activity, provides information about the target domain. It is the basis for existence of prior knowledge and forms the platform for its further evolvement and progression. Hence, the above three concepts (existence, evolvement, and advancement) can be accomplished within the numerical context when procedural knowledge is supported with declarative knowledge (which is assumed prior) and learning is situated to practise and is mediated via conversation in a socio-interactive environment with two or more participants involved.

Ontologically, domain knowledge should be organised or managed in a way that aids construction. Two views associated with management or organisation of knowledge could be identified. Business management scientists understand knowledge management as the systematic process of finding, selecting, organising, distilling and presenting information in a way that improves an employee's comprehension in a specific area of interest. On the other hand, computer scientists conceived it as the organisation of knowledge repositories (databases), to allow for easy retrieval and exchange of the information stored (Li \& Masters, 2010). Also, Grundspenkis (2008) considers ontology as a knowledge structure; this definition not only reflects the domain concepts, but also the relations between them. These last two views or definitions align with one of the theories (i.e. CT) underpinning ACCAM and is hereby upheld. The foregoing position represents the ontological foundation of the metamodel, wherein knowledge organisation is understood as entailment structures. ACCAM therefore adopts ontology as composed of domain topics/concepts and their interrelations.

\subsection{Learning Content-The Domain Knowledge and Strategies}

Domain knowledge is a key knowledge type that is captured in a technology-enhanced tutoring system (see Murray, 1998; Grundspenkis, 2008; Woolf, 2009). A metamodel that is meant to shape the design of such learning environment should therefore capture domain knowledge as well as the strategies that will be deployed to teach it.

Accordingly, ACCAM assumed full meaning associated with learning content as conceived in CA. That is, ACCAM's learning content comprises of the domain knowledge (the focus of teaching and learning) and strategies deployed in the knowledge construction process. However, ACCAM adopts domain knowledge definition prescribed in CT on two grounds: one, $\mathrm{CT}$ is a systems theory and the metamodel is meant to shape a system that is in a technology-based learning environment; two, CT proposes a KR scheme that captures knowledge in such an environment, an aspect that is explicitly omitted in CA theory.

\subsection{The Knowledge Representation (KR) Scheme}

Murray (1998) argues that a technology-enhanced learning environment should capture knowledge of what to teach (domain knowledge) and how to teach (pedagogy knowledge). Grundspenkis (2008) claims three types of knowledge are captured, namely domain knowledge, pedagogy knowledge and learner's knowledge. However, Akin (2008) conceives knowledge to be either declarative (propositional) or procedural knowledge. The latter knowledge dimensions can be argued not to be determinate or definitive. From AI perspective for instance, VanLehn (1987) claims that such rigid classification "is notorious ... as a fuzzy, seldom useful differentiation" (cited in Murray, 1998). Hence, such classification should be dumped except in the context in which it has precise meaning (Murray, 1998).

Whatever the position upheld, KR scheme would be required to store each of the knowledge types. In ACCAM, knowledge comprises of domain, pedagogy and learner's knowledge. This aims to avoid complexity and to limit and clarify the knowledge types in the KR scheme of the proposed metamodel. ACCAM assumes domain knowledge to be both declarative and procedural knowledge. Hence, ACCAM embraces a KR scheme that reflects the structure of knowledge types within a tutoring system. It conceived the scheme as comprising of knowledge units' inter-relationship, the semantic meaning of the units and their relationship in order to enhance access to knowledge during the learning process. At implementation level, the KR scheme are captured as a set of rules in which the concepts are interrelated. In order to generalise the metamodel for technology-enhanced learning, the metamodel could adopt any other KR scheme, inasmuch as such a scheme will allow the integration of concepts, and establish a connection between them. 


\subsection{The Sequencing Strategies}

According to VanLehn (1987), human experts did not discover their knowledge or infer it; they learned it from a mentor, either in school or as an apprentice. Thence, a good mentor should carefully select tasks that appropriately fit into a student's current knowledge state. This is because the learning content that is properly sequenced, potentially maximises or enhances learning (Tedman \& Tedman, 2007). Also, learning content should not be sequenced in a randomly order, but carefully structured (VanLehn, 1987).

ACCAM aligns with the above because it is logically arguable. The pedagogic metamodel upholds the view that meaningful learning is achievable when learning content, feedback, hints etc., are structured to learners' needs at every stage of their learning process. Hence, ACCAM assumes a structured sequence. Such sequence allows knowledge to be presented in a manner that aids its construction. The metamodel adopts an integrated sequencing pattern suggested in table 1 above and takes into consideration VanLehn's (1987) argument, stated above. Thus, knowledge in ACCAM is conceived to be presented according to complexity and diversity, and tailored to the level and learning style of the learner which is constantly monitored through augmented conversation.

\subsection{Teaching \& Learning Methodology}

The methodology that a theory advocate defines how teaching and learning is accomplished. It is expected that every learning theory or framework should make known the method for attaining the state-of-knowing. For example, Scott's (2001a), Laurillard's (2002) and Heinze, Procter and Scott's (2007) frameworks - underpinned by the epistemological stance of CT-canvass conversation as medium of coming to know. In formulating ACCAM's methodological approaches, CT and CA learning approaches were synthesised. Methodologically, the metamodel assumes learning is undertaken through conversation (see Pask, 1976), but augmented in order to reveal the thought process of the learner towards effective learning experience. This assumption leans on the historical effectiveness of conversation and its wide use at several levels of education, from childhood education (see Li \& Masters, 2010) to higher education (see Laurillard, 2002). Li and Masters (2010, p. 245), for instance, argues favourably for conversation in childhood education, thus: “... young children can learn through experience, application, and conversation in community..., with peers, parents, teachers, and other adults, ...".

Laurillard (2002) embeds conversation in the framework she developed for higher education. However, conversation in the ACCAM is conceived as a stepwise or phase-wise approach and informed by the methods proposed by CA. This ACCAM's methodological model aims to enhance construction of transferable knowledge. Hence, in consideration of the significance, relevance and actualisation of deep and transferable knowledge, the metamodel augments the conversation (CT learning strategy) and the six methods proposed in CA (modelling, coaching, scaffolding/fading, articulation, reflection and exploration) as tools for actualising learning.

\subsection{The Socio-Context of Learning - The Learning Space and Participants}

Li and Masters (2010, p.245) provides clue to possible learning spaces where conversation could be deployed, thus: "... children can learn through .... conversation in community, physically or virtually, with peers, parents, teachers, and other adults, beyond the classroom and across the media." Despite Li and Masters (2010) focus on childhood education, the learning spaces mentioned and participants involved in a learning process are equally applicable to adults at higher education. This is evidence in several frameworks implemented in higher education (Laurillard, 2002; Heinze, Procter, \& Scott, 2007). The stated implementations point to the imperative and relevance of the sociological context of learning.

Thus, ACCAM builds on the learning environment of its underlying theories. It proposes a socio-learning space involving two or more participants (consisting of learners or peers, parents, teachers and others engaged in conversation) and a learning space (wherein teaching and learning is phased into three stages). Such a learning space includes; at least, a domain expert or experienced person that directs the learning activities. ACCAM builds on CT and CA strategy, the need for at least a domain expert, experienced person or master, who helps coordinate teaching and learning (see Pask, 1988; Collins, Brown, \& Newman, 1989). Figure 3 below is a pictorial representation of the proposed metamodel's learning space. It provides insight into the socio- or learning-interaction occurring among the participants within the learning space. 


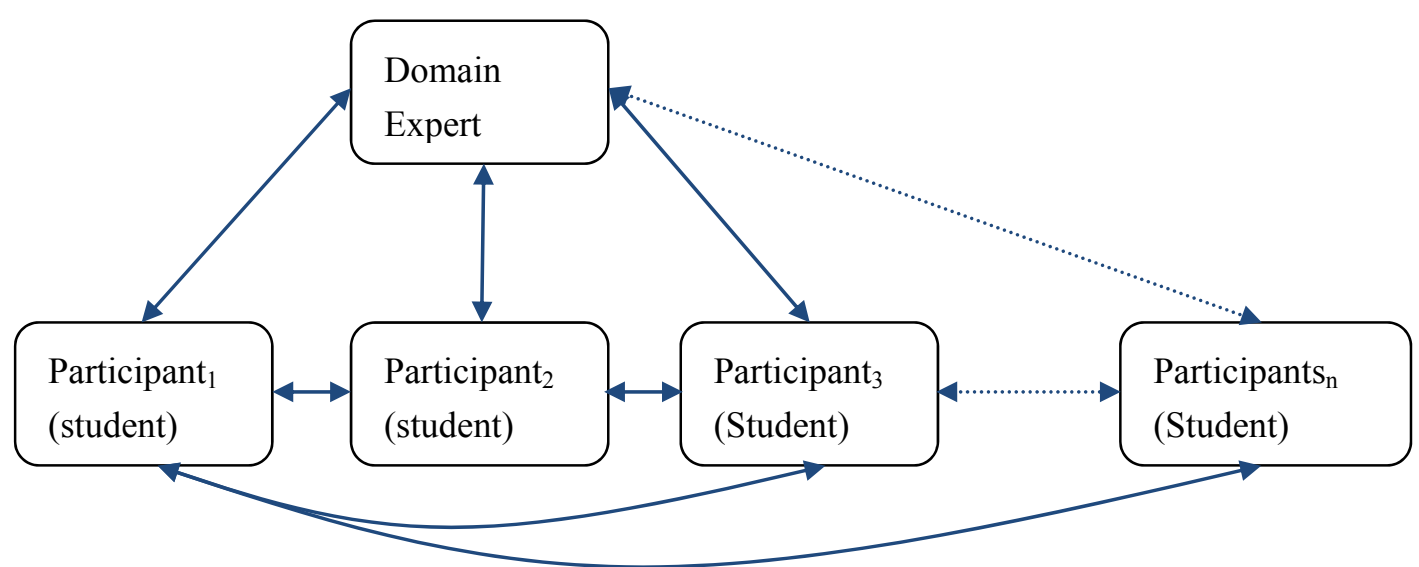

Figure 3. The ACCAM's socio-interaction learning space

\section{Prototyping ACCAM}

The theoretical elements of ACCAM foreshadow some design implications when implemented. The discussion in this section therefore throws light on the implications of ACCAM's theoretical elements with respect to the design of an ITS authoring tool. It elucidates how the former metamorphoses into the latter. Figure 4 below further illustrates the relationship between the ACCAM, ITS authoring tool (also known as ILABS in this paper) and its product (i.e. ITS). While the figure demonstrates the link between ILABS and its theoretical foundation (the ACCAM), it also signposts the three purposes of ILABS with respect to ITS construction i.e. to build a new ITS, modify an existing ITS, or extend an existing ITS. Consequently, the theoretical elements of ACCAM can be embedded in the ITS constructed through the ILABS. The following subsections therefore present the transformation of the theoretical elements into design features in ILABS.

\subsection{The Design Implications of the Theoretical Assumptions}

Conceptually, ACCAM assumes that conversation enhances cognitive visibility. The assumption is aimed at the generation of relevant feedbacks to enhance learning. This assumption (consisting of two concepts - conversation and cognitive visibility) informs the inclusion of a virtual calculator feature and three optional tutoring strategies (model-tracing, process monitoring, and no tutoring) in ILABS. The calculator is considered a key tool in a numerate problem-solving domain in which ACCAM is domicile. Any of the tutoring strategies can be optionally embedded in an ITS constructed through the ILABS.

Model-tracing strategy is a goal-oriented knowledge tracing strategy. It compares a learner's solution to a problem-solving goal of the domain expert. Process monitoring compares each cognitive step or node with that of a domain expert during a problem-solving process. These two strategies implement goal and step-wise tracking models respectively with appropriate feedback (see detail discussion of feedback types in VanLehn, 2006). However, the "No Tutoring" strategy encourages a student to explore a given problem without any guidance. Feedback is only provided at the end of the learning process, when completed work is submitted for marking. While "No tutoring" strategy implements a summative assessment, the other two strategies carry out formative assessment-but at differing levels. The "No tutoring" strategy enables the evaluation of the conceptual knowledge/skill a student has acquired over time. In addition, model tracing and process monitoring evaluate the gradual development of knowledge/skill at goal and step levels respectively.

The conception and implementation of tutoring strategies derived its theoretical foundation from ACCAM. The metamodel provides a strong educational foundation to evaluate educational research issues. This contrasts with previous cognitive or AI-based works that test cognitive/AI models or techniques in educational domain (e.g. Aleven, McLaren, Roll, \& Koedinger, 2006c; Zarandi, Khademian, \& Minaei-Bidgoli, 2012). Also, current work extends the implementation of model-tracing concept. It augments the existing conversation-based system (Kinshuk, Patel, \& Russell, 2000; Patel, Kinshuk, \& Rusell, 2000) through the introduction of the cognitive visibility concept.

The conversation-cognitive visibility abstraction is conceived as process monitoring which is implemented through an interactive medium - a calculator. The medium enables interaction between the learner and the tutoring system generated through ILABS. The foregoing implementation provides the platform to monitor learner's cognitive process during a numerical problem solving activity. Impliedly, process monitoring 
implementation requires an improvised virtual calculator, unlike the other two strategies (i.e model-tracing and no-tutoring strategies) - in which the calculator is optional. The embedded strategies translate into different tutoring behaviours in the constructed ITS during learning.

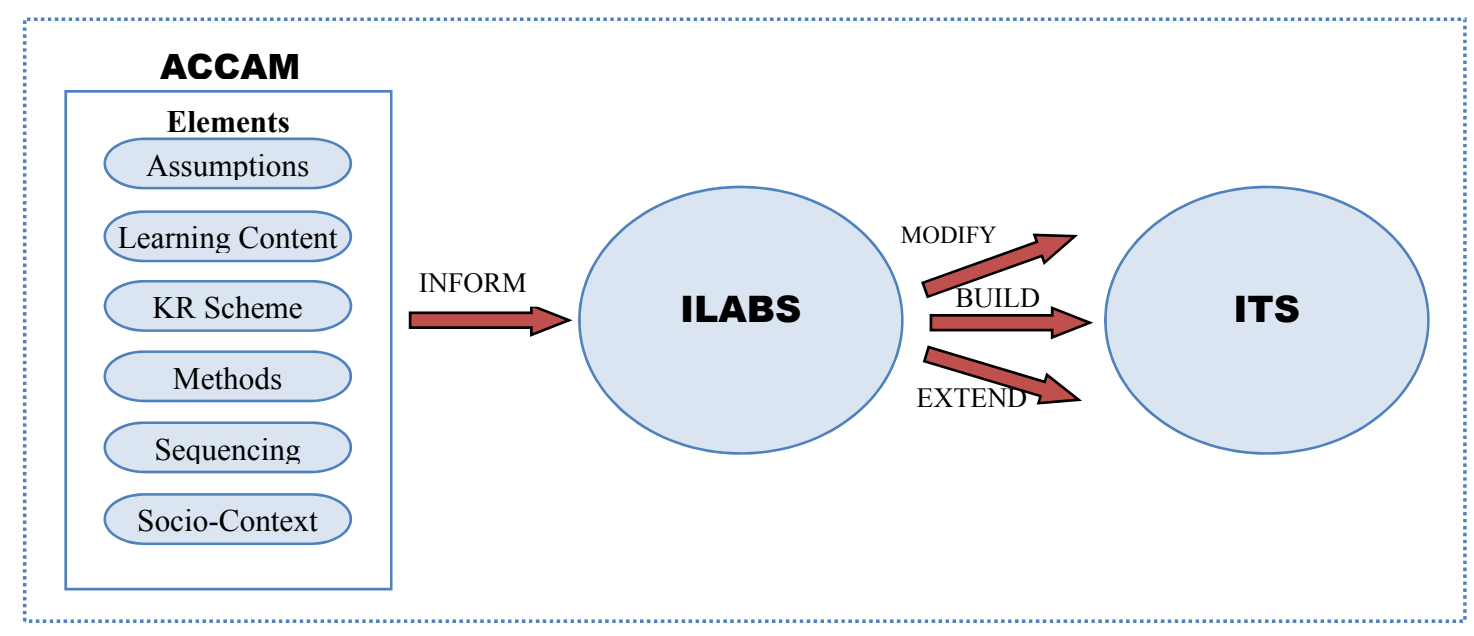

Figure 4. ACCAM's implementation model

\subsection{The Design Implications of Learning Content / Knowledge Representation Elements}

Learning content represents the knowledge of the subject or topic of a target domain. Since research is undertaken in context (Luckin, 2010), ACCAM is domiciled in numerate problem-solving context. The ACCAM's learning content covers numerical topics within numerical disciplines. These include; the numerical aspects of the engineering, accounting and finance, actuarial science and other applied domains that involve categorisation and/or application of rules. The ACCAM domain-driven conception provides the basis to evaluate and eventually generalise the metamodel for implementation in ITS authoring tools. This conceptual approach aligns with trends in the literature, in which new strategies or research issues are tested in specific domain(s) before generalisation (e,g. Chi, VanLehn, Litman, \& Jordan, 2011; Gilbert et al., 2011; Dewan, 2012). Murray (1999) argues that the applicability of an ITS authoring tool may not be feasible for all possible domains; it should be limited to certain knowledge types because it would enable the production of usable and powerful ITSs. Hence, the reason for constraining ACCAM's within the numerical disciplines.

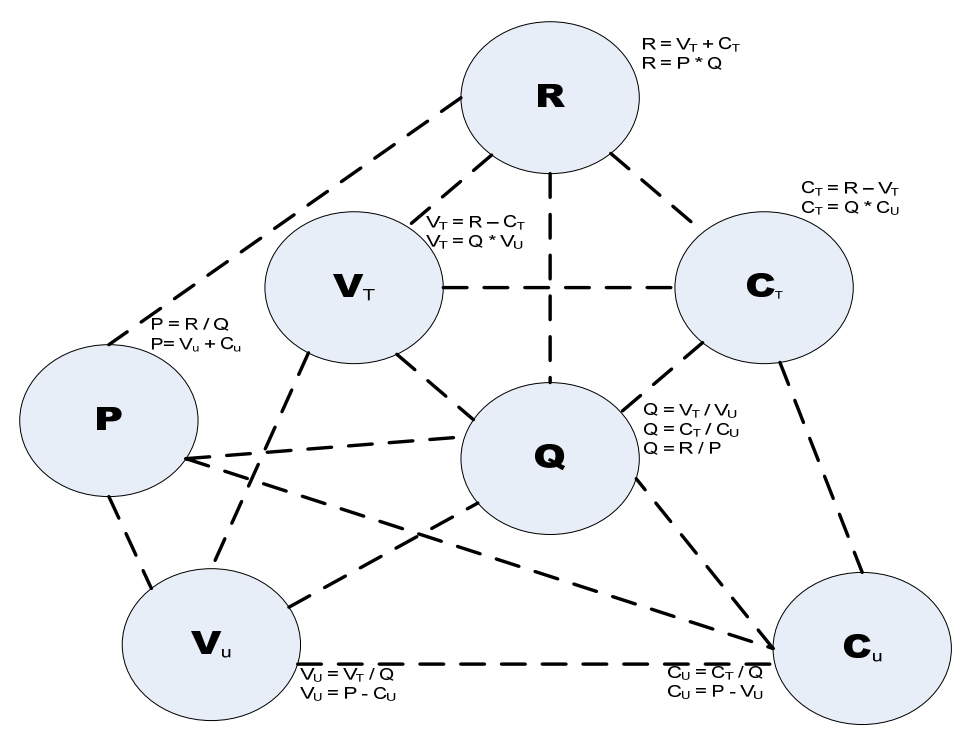

Source: adopted from Patel and Kinshuk (1997).

Figure 5. Network of interrelated nodes of domain knowledge 
Also, the design implication of the KR scheme of the ACCAM assumes the learning content (i.e. the domain knowledge) as a set of nodes (or units) in a network of interconnected relationships. Current work conceives the implementation of the learning content in fine grain detail to enhance both goal and step-wise tracing of knowledge. This contrasts to Patel and Kinshuk (1997)'s only goal-tracing implementation — see Figure 5 above that describes 7 partial networks of 14 variables involved in solving problems in marginal costing based upon the following equations:

$$
\begin{aligned}
& \mathrm{R}=\mathrm{Q} * \mathrm{P} \rightarrow \text { Revenue }=\text { Quantity }^{*} \text { Price } \\
& \mathrm{V}_{\mathrm{T}}=\mathrm{Q} * \mathrm{~V}_{\mathrm{U}} \rightarrow \text { Variable }_{(\text {total) }} \text { cost }=\text { Quantity }^{*} \text { Variable }_{\text {(unit) }} \text { cost } \\
& \mathrm{C}_{\mathrm{T}}=\mathrm{Q} * \mathrm{C}_{\mathrm{U}} \rightarrow \text { Contribution }_{(\text {total) }}=\text { Quantity }^{*} \text { Contribution }_{(\text {unit) }} \\
& \mathrm{C}_{\mathrm{T}}=\mathrm{R} * \mathrm{~V}_{\mathrm{T}} \rightarrow \text { Contribution }_{(\text {total) }}=\text { Revenue }^{*} \text { Variable }_{(\text {total) }} \text { cost }
\end{aligned}
$$

A unit or node can be an arithmetic operator (or any other operand e.g. “+”, ““_, “*”, etc.), a variable (regarded as a container that holds a value e.g. cost, sales, quantity etc.) and a predefined or imported function(s). All the nodes - variables, operands, functions - and the relationship (i.e. operators) that exist between them thus represents the domain knowledge (i.e. the learning content). Note that the domain knowledge domiciles in the domain module of an ITS, one of the four structures that an ITS authoring tool should construct.

\subsection{The Design Implication of the Teaching-Learning Methods}

The design implementation of ACCAM conceptualises teaching and learning via conversation and cognitive visibility, identification of misconception and missing conceptions, scaffolding and fading of guidance. The conversation is aimed at the enhancement of cognitive visibility - as earlier argued above. A conversation is an information exchange (Klemm, 2002; Holland \& Childress, 2008). It involves bidirectional communication and interactive exchanges between participants. Conversation could be verbal or non-verbal (Sharples, 2005; Scott \& Cong, 2010; Graesser \& D’Mello, 2012). The metamodel assumes the latter type of conversation and involves at least two participants - the learner and the tutoring system (that provides feedbacks and houses the expert knowledge within the domain module).

The ACCAM informs the need for a set of generic algorithms that should constitute the underlying procedures of an ITS authoring tool. These algorithms are embedded in an ITS depending on the tutoring strategies configuration during the authoring process. The algorithms implement the various aspects of teaching and learning, such as conversation, cognitive visibility, scaffolding, fading, etc. Note that conversation is regarded as the key to the implementation of other teaching and learning functionalities in the ACCAM.

\subsection{The Design Implications of the Sequencing Strategy}

The design of template-like features in an ITS authoring tool was conceived to implement the sequencing strategy. This would enable the configuration of problem templates with different levels of complexity and diversity. As earlier argued, the domain content should not be in a randomly ordered sequence, but a carefully structured one (VanLehn, 1987). In order to enhance the structuring of domain knowledge, problem templates are code named, and assigned complexity and diversity codes in an ascending level of complexity-from the least complex to the most difficult problem.

The implementation of problems, complexities and diversities require, in the first instance, the creation of problem diversity group names. This is followed by complexity groups for each diversity group. Note that for domains not requiring two or more diversity groups, complexity groups with their respective problem templates constitute a single diversity group. The implementation of the metamodel in an ITS authoring tool should allow authors (i.e. lecturers) to specify how the constructed ITS generates problems during learning. Problem complexity and diversity are allotted either in a specified structured pattern, sequentially or randomly. However, problems are randomly generated for each pair of complexity and diversity group. In order to address the global and local strategies of the metamodel, each domain problem is globally presented; that is, all the variables required to address a specific topic are displayed on screen. A learner is then required to localise the displayed problem; he/she reflects on the relationship between the variables globally, thereafter, addresses them, one at a time, without any ordered sequence enforced.

\subsection{The Design Implications of the Socio-Context Element}

The sociological context of learning involves two or more participants shaping learning of a target domain. The socio-context learning space consists of learners (peers), parents, teachers and others, as earlier mentioned. This learning space must include a domain expert or experienced person to coordinate the learning process. However, current work assumes a micro-implementation of the socio-context where two participants are involved viz; the 
learner and the machine (the expert system) that interprets and shapes the learning process of the learner.

The socio-context implementation thus requires that the ACCAM's knowledge representation scheme captures the domain knowledge and rules in an ITS authoring tool. Thereafter, interactive screen(s) (that is linked to captured domain knowledge) is/are configured. Each screen represents the medium of interaction between the learner and the machine or tutoring system. The learning space enables the learner (the first participant) to engage the tutoring system and, in response, the tutoring system (acting as the second participant) interprets the learning actions to provide guidance to the learner. Through the foregoing process, a micro socio-context learning space is provided. This results in a traditional one-to-one tutoring environment, wherein a more experienced person coordinates a novice in learning a target subject.

\section{Summary}

This work attempts to address a fundamental research goal, the provision of a priori link between theory and an ITS authoring tool. This research goal was addressed by proposing a pedagogic metamodel that derives and advances theoretical elements from two learning theories. Each of the underpinning theories was reviewed. The conception of the pedagogic metamodel - the ACCAM — upholds many of the characteristics of its underlying theories.

The ACCAM represents a synergistic framework. It advances the existing theoretical frameworks through the introduction of the concept of "augmentation". The implementation of ACCAM provides a practical and easy-to-use authoring environment that captures the characteristics of the metamodel, and enables authoring by teachers (or lecturers) without programming skills. Since this work represents a conceptual element of the research being reported, results of the evaluation of the intended authoring tool, that showcases practical reality of the metamodel discussed, is not considered in this paper. However, the evaluation is considered for future work.

\section{Future Work}

As earlier discussed, ACCAM represents a design approach informed by two pedagogical theories of teaching and learning. It therefore becomes imperative to evaluate its potential success as claimed in this paper. In future work, a report of the methodological approach for the evaluation of ACCAM is intended. Result of that evaluation which involves quantitative and qualitative evaluation will be published. Also, extended work on the implementation of the collaborative element of ACCAM is planned. Consideration of affect issues in ACCAM is also being considered because of its vital role in teaching and learning process.

\section{Acknowledgement}

This paper discusses one of the outputs of a research work that was part-funded by the De Montfort University, UK, through the Teaching and Learning Fund. The authors wish to thank all those who made it feasible.

\section{References}

Adenowo, A. A. A. (2012). Augmented Conversation and Cognitive Apprenticeship Metamodel Based Intelligent Learning Activity Builder System. PhD Thesis, De Montfort University, UK.

Akin, O. (1986). Psychology of Architectural Design. Pion Ltd., London.

Akin, O. (2008). Frames of Reference in Architectural Design: Analyzing the Hyper-Acclamation (A-h-a-!). School of Architecture, Paper 25, 1-20. Retrieved from http://repository.cmu.edu/architecture/25

Aleven, V., Sewall, J., McLaren, B. M., \& Koedinger, K. R. (2006a). Rapid Authoring of Intelligent Tutors for Real-World and Experimental Use. In K. Kinshuk, R. Koper, P. Kommers, P. Kirschner, D. G. Sampson \& W. Didderen (Eds.), Sixth IEEE International Conference on Advanced Learning Technologies (ICALT'06) (pp. 847-851). Los Alamitos, CA: IEEE Computer Society. http://dx.doi.org/10.1109/ICALT.2006.1652575

Aleven, V., McLaren, B., Sewall, J., \& Koedinger, K. (2006b). The cognitive tutor authoring tools (ctat): Preliminary evaluation of efficiency gains. In M. Ikeda, K. D. Ashley \& T. W. Chan (Eds.), Proceedings of the 8th International Conference on Intelligent Tutoring Systems (pp. 61-70). Berlin: Springer Verlag: Springer. http://dx.doi.org/10.1007/11774303_7

Aleven, V., McLaren, B., Roll, I., \& Koedinger, K. (2006c). Toward meta-cognitive tutoring: A model of help seeking with a Cognitive Tutor. International Journal of Artificial Intelligence in Education, 16(2), 101-128. IOS Press.

Blessing, S. B., Gilbert, S. B., Ourada, S., \& Ritter, S. (2009). Authoring Model-Tracing Cognitive Tutors. International Journal of Artificial Intelligence in Education, 19(2), 189-210. IOS Press. 
Boyd, G. M. (2004). Conversation theory. In D. D. Jonassen (Ed.), Handbook of research on educational communications and technology (2nd ed., pp. 179-198). Mahwah, NJ: Lawrence Erlbaum Associates.

Brown, J. S., Collins, A., \& Duguid, P. (1989). Situated Cognition and the Culture of Learning. Educational Researcher, 18(1), 32-42. http://dx.doi.org/10.3102/0013189X018001032

Brusilovsky, P. (2003). Developing adaptive educational hypermedia systems: From design models to authoring tools. In T. Murray, S. Blessing, \& S. Ainsworth (Eds.), Authoring tools for advanced technology Learning Environments (pp. 377-409). London: Kluwer Academic Publishers. http://dx.doi.org/10.1007/978-94-017-0819-7_13

Chi, M., VanLehn, K., Litman, D., \& Jordan, P. (2011). An Evaluation of Pedagogical Tutorial Tactics for a Natural Language Tutoring System: A Reinforcement Learning Approach. International Journal of Artificial Intelligence in Education, 21, 83-113.

Collins, A., Brown, J. S. \& Holum, A. (1991). Cognitive apprenticeship: Making thinking visible. American Educator, 15(3), 6-11.

Collins, A., Brown, J. S., \& Newman, S. E. (1989). Cognitive Apprenticeship: Teaching the Crafts of Reading, Writing, and Mathematics. In L. B. Resnick (Ed.), Knowing, Learning, and Instruction: Essays in honor of Robert Glaser (pp. 453-494). Hillsdale, NJ: Lawrence Erlbaum Associates Inc.

Dennen, V. P. (2004). Cognitive apprenticeship in educational practice: Research on scaffolding, modeling, mentoring, and coaching as instructional strategies. In D. H. Jonassen (Ed.), Handbook of research on educational communications and technology (2nd ed., pp. 813-828). Lawrence Erlbaum Associates Mahwah, NJ.

Dennen, V. P., \& Burner, K. J. (2008). The Cognitive Apprenticeship Model in Educational Practice. In J. M. Spector, M. D. Merrill, J. van Merrienboer \& M. P. Driscoll (Eds.), Handbook of research on educational communications and technology (3rd ed., pp. 425-439). New York: Lawrence Erlbaum Associates.

Dewan, P. (2012). How a language-based GUI generator can influence the teaching of object-oriented programming. Proceedings of the 43rd ACM technical symposium on Computer Science Education (pp. 69-74).

Gilbert, S., Devasani, S., Kodavali, S., \& Blessing, S. (2011). Easy Authoring of Intelligent Tutoring Systems for Synthetic Environments. Proceedings of the Twentieth Conference on Behavior Representation in Modeling and Simulation.

Graesser, A. C., Conley, M. W., \& Olney, A. (2012). Intelligent Tutoring Systems. In K. R. Harris, S. Graham \& T. Urban (Eds.), APA Educational Psychology Handbook (Vol. 3, Chap. 19: Application to Teaching and Learning, pp. 451-473).

Graesser, A. C., \& D'mello, S. (2012). Affect-Sensitive Intelligent Tutoring System. US Patent 2012/0052476 A1. Retrieved from http://www.freepatentsonline.com/y2012/0052476.html

Grundspenkis, J. (2008). Knowledge Creation Supported by Intelligent Knowledge Assessment System. Proceedings of the 12th World Multi-Conference on Systemics, Cybernetics and Informatics, June 29-July 2, 2008 (pp. 135-140). Orlando, Florida, USA.

Hartley, R. (2010). The evolution and redefining of "CAL": a reflection on the interplay of theory and practice. Journal of Computer Assisted Learning, 26(1), 4-17. http://dx.doi.org/10.1111/j.1365-2729.2009.00340.x

Hayashi, Y., Bourdeau, J., \& Mizoguchi, R. (2009). Using ontological engineering to organize learning/instructional theories and build a theory-aware authoring system. International Journal of Artificial Intelligence in Education, 19(2), 211-252. IOS Press.

Heinze, A., Procter, C., \& Scott, B. (2007). Use of conversation theory to underpin blended learning. International Journal of Teaching and Case Studies, 1(1/2), 108-120. http://dx.doi.org/10.1504/IJTCS.2007.014213

Holland, J., \& Childress, M. (2008). Conversation Theory Conceptualized in E-Learning Environments (pp. 80-89). IGI Global.

Klemm, W. (2002). Software issues for applying conversation theory for effective collaboration via the Internet. Proceedings of the 2002 International Conference. Retrieved from $\mathrm{http} / /$ scholar.google.com/scholar?hl=en\&btnG=Search\&q=intitle:Software+Issues+for+Applying+Convers ation+Theory+For+Effective+Collaboration+Via+the+Internet $\# 0$ 
Laurillard, D. (2002). Rethinking University Teaching - a framework for the effective use of educational technology. London: Routledge.

Li, D., Zhuying, L., \& Bing, Y. (2010). The Application of AI Technology in Intelligent Tutoring System. $20102^{\text {nd }}$ International Conference on Education Technology and Computer (ICETC), China (Vol. 1, pp. 1-490).

Li, H., \& Masters, J. (2010). Editorial: E-learning and Knowledge Management in the Early Years: Where Are We and Where Should We Go. Knowledge Management \& E-Learning: An International Journal (KM\&EL), 1(4), 245.

Longo, D. R., Katerndahl, D. A., Turban, D. B., Griswold, K., Ge, B., Hewett, J. E., ... Schubert, S. (2011). The Research Mentoring Relationship in Family Medicine: Findings From the Grant Generating Project. Family Medicine, 43(4), 240-247.

Luckin, R. (2010). Re-designing Learning Contexts: Technology-rich, Learner-Centred Ecologies. London: Routledge, Taylor \& Francis Group.

Matsuda, N., \& VanLehn, K. (2005). Advanced Geometry Tutor: An intelligent tutor that teaches proof-writing with construction. Proceedings of the 12th International Conference on Artificial Intelligence in Education (pp. 443-450). Amsterdam.

Mitrovic, A., Martin, B., \& Suraweera, P. (2009). ASPIRE: an authoring system and deployment environment for constraint-based tutors. International Journal of Artificial Intelligence in Education, 19, 155-188. IOS Press.

Mizoguchi, R., Hayashi, Y., \& Bourdeau, J. (2010). Ontology-Based Formal Modeling of the Pedagogical World: Tutors Modeling. In R. Nkambou, J. Bourdeau \& R. Mizoguchi (Eds.), Advances in Intelligent Tutoring Systems (pp. 229-248). Berlin Heidelberg: Springer-Verlag. http://dx.doi.org/10.1007/978-3-642-14363-2_11

Moundridou, M., \& Virvou, M. (2001). Authoring and delivering adaptive Web-based textbooks using WEAR. In T. Okamoto, R. Hartley, Kinshuk, \& J. P. Klus (Eds.), Proceedings IEEE International Conference on Advanced Learning Technologies; Issues, Achievements, and Challenges-ICALT 2001 (pp. 185-188). Los Alamitos, California: IEEE Computer Society.

Moundridou, M., \& Virvou, M. (2002). Evaluating the instructor support provided by a Web-based authoring tool for building adaptive courses. Proceedings of the IEEE International Conference on Advanced Learning Technologies-ICALT 2002 (pp. 408-413).

Moundridou, M., \& Virvou, M. (2003). Analysis and design of a web-based authoring tool generating intelligent tutoring systems. Computers \& Education, 40, 157-181. http://dx.doi.org/10.1016/S0360-1315(02)00119-7

Murray, T. (1998). Authoring Knowledge-Based Tutors: Tools for Content, Instructional Strategy, Student Model, and Interface Design. Journal of the Learning Sciences, 7(1), 5-64. http://dx.doi.org/10.1207/s15327809j1s0701_2

Murray, T. (1999). Authoring intelligent tutoring systems: An analysis of the state of the art. International Journal of Artificial Intelligence in Education, 10(1), 98-129.

Murray, T. (2003a). An Overview of Intelligent Tutoring System Authoring Tools: Updated Analysis Of The State Of The Art. In T. Murray, S. Blessing, \& S. Ainsworth (Eds.), Authoring Tools For Advanced Technology Learning Environments. Kluwer Academic Publishers. http://dx.doi.org/10.1007/978-94-017-0819-7_17

Murray, T. (2003b). EON: Authoring Tools for Content, Instructional Strategy, Student Model, and Interface Design. In T. Murray, S. Blessing \& S. Ainsworth (Eds.), AuthoringTools for Advanced Technology Learning Environment. Kluwer Academic Publishers. http://dx.doi.org/10.1007/978-94-017-0819-7_11

Nkambou, R., Bourdeau, J., \& Mizoguchi, R. (2010). Introduction: What are Intelligent Tutoring Systems, and Why This Book? In R. Nkambou, J. Bourdeau \& R. Mizoguchi (Eds.), Advances in Intelligent Tutoring Systems. Berlin Heidelberg: Springer-Verlag (pp. 1-14). http://dx.doi.org/10.1007/978-3-642-14363-2_1

Nkambou, R., Frasson, C., \& Gauthier, G. (2003). CREAM-TOOLs: An Authoring Environment for Knowledge Engineering in Intelligent Tutoring Systems. In T. Murray, S. Ainsworth \& S. Blessing (Eds.), Authoring Tools for Advance Technology Learning Environments (pp. 269-308). http://dx.doi.org/10.1007/978-94-017-0819-7_10

Ohlsson, S., \& Mitrovic, A. (2006). Constraint-based knowledge representation for individualized instruction. Computer Science and Information Systems, 3(1), 1-22. http://dx.doi.org/10.2298/CSIS0601001S 
Pask, G. (1975). Conversation, Cognition and Learning. Amsterdam and New York: Elsevier.

Pask, G. (1976). Conversational techniques in the study and practice of education. British Journal of Educational Psychology, 46(1), 12-25. Wiley Online Library. http://dx.doi.org/10.1111/j.2044-8279.1976.tb02981.x

Pask, G. (1988). Learning strategies, teaching strategies, and conceptual or learning style. In R. D. Schmeck (Ed.), Learning strategies and learning styles (pp. 83-99). New York: Plenum Publishing Corporation. http://dx.doi.org/10.1007/978-1-4899-2118-5_4

Pask, G., Scott, B., \& Kallikourdis, D. (1973). A theory of conversations and individuals exemplified by the learning process on CASTE. Int. Journal of Man-Machine Studies, 5, 443-566. http://dx.doi.org/10.1016/S0020-7373(73)80002-1

Patel, A., \& Kinshuk. (1997). Intelligent Tutoring Tools in a Computer-Integrated Learning Environment for Introductory Numeric Disciplines. Innovations in Education and Teaching International, 34(3), 200-207. http://dx.doi.org/10.1080/1355800970340306

Patel, A., Kinshuk, K., \& Russell, D. (2000). Intelligent Tutoring Tools for Cognitive Skill Acquisition in Life Long Learning. Educational Technology \& Society, 3(1), 32-40.

Patel, A., \& Russell, D. (2000). A multi-institutional evaluation of Intelligent Tutoring Tools in Numeric Disciplines. Educational Technology \& Society, 3(4), 66-74.

Scott, B. (2001a). Conversation Theory: A Constructivist, Dialogical Approach to Educational technology. Cybernetics \& Huamn Knowing, 8(4), 25-46.

Scott, B. (2001b). Gordon Pask's conversation theory: A domain independent constructivist model of human knowing. Foundations of Science, 6(4), 343-360. http://dx.doi.org/10.1023/A:1011667022540

Scott, B. (2007). "The Cybernetics of Gordon Pask", in Gordon Pask, Philosopher Mechanic: An Introduction to the Cybernetician. In R. Glanville \& K. H. Muller (Eds.), editionechoraum, WISDOM, Vienna (pp. 29-52).

Scott, B., \& Cong, C. (2008). "Analysing and representing "conversational domains"” Proceeding of the $19^{\text {th }}$ European Meeting on Cybernetics and Systems Research (pp.132-137). University of Vienna, March 2th-28th, 2008.

Scott, B., \& Cong, C. (2010). Evaluating course design principles for multimedia learning materials. Campus-Wide Information Systems, 27(5), 280-292. http://dx.doi.org/10.1108/10650741011087720

Self, J. (1990). Theoretical foundations for intelligent tutoring systems. Journal of Artificial Intelligence in Education, 1(4), 3-14.

Self, J. (1994). Formal approaches to student modelling. In G. I. McCalla \& J. Greer (Eds.), Student Modelling: the key to individualized knowledge-based instruction (pp. 295-352). Berlin: Springer-Verlag. http://dx.doi.org/10.1007/978-3-662-03037-0_12

Self, J. (1999). The defining characteristics of intelligent tutoring systems research: ITSs care, precisely. International Journal of Artificial Intelligence in Education, 10(3-4), 350-364.

Sharples, M. (2005). Learning as conversation: Transforming education in the mobile age. Proceedings of Conference on Seeing, Understanding, Learning in the Mobile Age (pp. 147-152).

Tedman, R. \& Tedman, D. (2007). Introduction to the evolution of teaching and learning paradigms. Evolution of Teaching and Learning Paradigms in Intelligent Environment, Studies in Computational Intelligence, 62, 1-6. Springer. http://dx.doi.org/10.1007/978-3-540-71974-8_1

VanLehn, K. (1987). Learning One Subprocedure per Lesson. Artificial Intelligence, 31(1), 1-40. http://dx.doi.org/10.1016/0004-3702(87)90080-4

VanLehn, K. (2006). The behavior of tutoring systems. International Journal of Artificial Intelligence in Education, 16(3), 227-265.

Virvou, M., \& Moundridou, M. (2000). A web-based authoring tool for algebra-related intelligent tutoring systems. Educational Technology \& Society, 3(2), 61-70.

von Glasersfeld, E. (2002). Cybernetics and the Theory of Knowledge. UNESCO Encyclopedia. Retrieved from http://www.vonglasersfeld.com/255

Woolf, B. P. (2009). Building intelligent interactive tutors: Student-centered strategies for revolutionizing e-learning (pp. 1-467). San Francisco, CA: Elsevier Inc., Morgan Kauffman. 
http://dx.doi.org/10.1016/B978-0-12-373594-2.00001-0

Zarandi, M. H. F., Khademian, M., \& Minaei-Bidgoli, B. (2012). A Fuzzy Expert System Architecture for Intelligent Tutoring Systems: A Cognitive Mapping Approach. Journal of Intelligent Learning Systems and Applications, 04(01), 29-40. http://dx.doi.org/10.4236/jilsa.2012.41003

\section{Copyrights}

Copyright for this article is retained by the author(s), with first publication rights granted to the journal.

This is an open-access article distributed under the terms and conditions of the Creative Commons Attribution license (http://creativecommons.org/licenses/by/3.0/). 